TRANSACTIONS OF THE

AMERICAN MATHEMATICAL SOCIETY

Volume 352, Number 11, Pages 5251-5262

S 0002-9947(00)02547-2

Article electronically published on July 18, 2000

\title{
OPTIMAL FACTORIZATION OF MUCKENHOUPT WEIGHTS
}

\author{
MICHAEL BRIAN KOREY
}

\begin{abstract}
Peter Jones' theorem on the factorization of $A_{p}$ weights is sharpened for weights with bounds near 1 , allowing the factorization to be performed continuously near the limiting, unweighted case. When $1<p<\infty$ and $w$ is an $A_{p}$ weight with bound $A_{p}(w)=1+\varepsilon$, it is shown that there exist $A_{1}$ weights $u, v$ such that both the formula $w=u v^{1-p}$ and the estimates $A_{1}(u), A_{1}(v)=1+\mathcal{O}(\sqrt{\varepsilon})$ hold. The square root in these estimates is also proven to be the correct asymptotic power as $\varepsilon \rightarrow 0$.
\end{abstract}

\section{INTRODUCTION}

A non-negative weight function $w$ on $\mathbb{R}^{n}$ is in the Muckenhoupt $A_{p}$ class, $w \in A_{p}$, if there is a constant $C$ such that

$$
\left(\frac{1}{|Q|} \int_{Q} w\right)\left(\frac{1}{|Q|} \int_{Q} w^{1 /(1-p)}\right)^{p-1} \leq C
$$

for all cubes $Q$ in $\mathbb{R}^{n}$ with sides parallel to the coordinate axes. Here and throughout this note $|Q|$ denotes the Lebesgue measure of $Q$, integrals are evaluated with respect to Lebesgue measure, and $1<p<\infty$. The smallest constant $C$ for which (11) holds is termed the $A_{p}$ bound of $w$ and is denoted $A_{p}(w)$; note that $A_{p}(w) \geq 1$, by Hölder's inequality, with equality only when $w$ is almost everywhere constant. The limiting case $w \in A_{1}$ is defined by the requirement that

$$
\frac{1}{|Q|} \int_{Q} w \leq C \inf _{Q} w
$$

for all cubes $Q$, where $\inf _{Q} w$ denotes the essential infimum of $w$ over $Q 11$ The least bound $C$ in (2), denoted $A_{1}(w)$, is likewise at least 1.

Products of suitable powers of $A_{1}$ weights are in $A_{p}$. In fact, if $u$ and $v$ are in $A_{1}$, then $u v^{1-p}$ is in $A_{p}$, and the bound of this product satisfies the estimate

$$
A_{p}\left(u v^{1-p}\right) \leq A_{1}(u) A_{1}(v)^{p-1},
$$

as follows directly from conditions (1) and (2). By means of a delicate stoppingtime argument, Jones 6] succeeded in proving the converse: each $A_{p}$ weight $w$ can be decomposed as the product $w=u v^{1-p}$ of $A_{1}$ weights $u$ and $v$. Several years later, Rubio de Francia found a much simpler proof of this decomposition (see [2], [12], and [13]), and his "reiteration" scheme has since found many applications. It

Received by the editors February 3, 1999.

1991 Mathematics Subject Classification. Primary 42B25; Secondary 26D15, 46E30.

Key words and phrases. Jones' factorization theorem, bounded mean oscillation, vanishing mean oscillation, $A_{p}$ condition.

${ }^{1}$ In general, all pointwise statements in this paper should be understood to hold only almost everywhere with respect to Lebesgue measure. 
has been used, for example, to give a constructive proof of the duality of Hardy space $H^{1}$ and BMO, the space of functions of bounded mean oscillation (see [3]); to prove an extrapolation theorem for operators on weighted $L^{p}$ spaces (see [12]); and to characterize the domains on which BMO functions have extensions to all $\mathbb{R}^{n}$ (see 5]).

For the purpose to be discussed here, however, the reiteration argument has one shortcoming: it does not give sharp quantitative information on the weight bounds $A_{1}(u)$ and $A_{1}(v)$ of the factors that arise in the decomposition of a given $A_{p}$ weight $w$ as $u v^{1-p}$. In particular, it does not reveal whether it is possible to factor $A_{p}$ weights with bounds near 1 "continuously" into pairs of component weights with $A_{1}$ bounds near 1 . By contrast, the estimate 3 immediately shows that when the bounds $A_{1}(u)$ and $A_{1}(v)$ are near 1 , then so is $A_{p}\left(u v^{1-p}\right)$.

To see how this difficulty arises, let us briefly review the reiteration argument in the simplest case $p=2$, in which we seek to factor a given $A_{2}$ weight $w$ into a quotient of two $A_{1}$ weights (see [15]).

Since $w \in A_{2}$, the Hardy-Littlewood maximal operator $M$ is bounded both on $L^{2}(w d x)$ and, by the symmetry in (1), on $L^{2}\left(w^{-1} d x\right) 2$ It follows that the sublinear operator $S$ defined by

$$
S(f)=w^{-1 / 2} M\left(w^{1 / 2} f\right)+w^{1 / 2} M\left(w^{-1 / 2} f\right)
$$

is bounded on the unweighted space $L^{2}(d x)$, say $\|S(f)\|_{2} \leq B\|f\|_{2}$. Now choose a positive function $f$ in $L^{2}(d x)$, as well as a number $\lambda$ larger than 1 . Next, set $g=\sum_{k=1}^{\infty}(\lambda B)^{-k} S^{k}(f)$. Then $g \in L^{2}(d x)$ and

$$
S(g)=(\lambda B) \sum_{k=2}^{\infty}(\lambda B)^{-k} S^{k}(f)=(\lambda B) g-S(f) .
$$

Since $S(f) \geq 0$, the pointwise estimate $S(g) \leq(\lambda B) g$ holds. Thus

$$
w^{-1 / 2} M\left(w^{1 / 2} g\right) \leq S(g) \leq(\lambda B) g
$$

so that $M\left(w^{1 / 2} g\right) \leq \lambda B\left(w^{1 / 2} g\right)$. Hence $u=w^{1 / 2} g$ belongs to $A_{1}$ and satisfies $A_{1}(u) \leq \lambda B$. Similarly, $v=w^{-1 / 2} g$ is in $A_{1}$, and $A_{1}(v) \leq \lambda B$. The construction thus quickly decomposes $w$ as a quotient $u / v$ of two $A_{1}$ weights; it does not, however, sharply control the $A_{1}$ bounds of the factors in this quotient. For even if the $A_{2}$ bound of the original weight $w$ is near 1 , we can only conclude from the above argument (letting $\lambda$ approach 1) that the $A_{1}$ bounds of $u$ and $v$ are no larger than the operator bound $B$, and this is at least 23

Thus, the reiteration scheme, while useful in numerous applications, does not answer the question we pose here: if $A_{2}(w)$ is near 1 , then is it possible to factor $w$ as a quotient of two $A_{1}$ weights $u$ and $v$ with bounds also near 1 ? The affirmative answer to this question is contained in the following theorem, the proof of which is the focus of this paper.

\footnotetext{
${ }^{2}$ Recall that $M f\left(x_{0}\right)=\sup _{Q}(1 /|Q|) \int_{Q}|f|$, where the supremum runs over all cubes $Q$ containing $x_{0}$. For the fundamental proof that $M$ is bounded on the weighted space $L^{2}(w d x)$ exactly when $w \in A_{2}$, see [1] or [10.

${ }^{3}$ Simply observe that for $f$ positive, both $M\left(w^{1 / 2} f\right) \geq w^{1 / 2} f$ and $M\left(w^{-1 / 2} f\right) \geq w^{-1 / 2} f$, so that $S(f) \geq 2 f$. More significantly, the norm of each of the two terms in $S$, viewed as an operator on $L^{2}(d x)$, is strictly greater than 1 .
} 
Theorem. If $w$ is an $A_{p}$ weight and $A_{p}(w)=1+\varepsilon<1+\varepsilon_{0}$, then there exist $A_{1}$ weights $u$ and $v$ satisfying both $w=u v^{1-p}$ and

$$
A_{1}(u) \leq 1+C \sqrt{\varepsilon}, \quad A_{1}(v) \leq 1+C \sqrt{\varepsilon} .
$$

The constants $C$ and $\varepsilon_{0}$ depend only on the dimension $n$ and the index $p$.

The method of the proof is first to supplement the original argument of Jones [6] in the dyadic model case with some sharp estimates in the author's thesis 8. The averaging method of Garnett and Jones [4] is then adapted to handle the general case. Sharpness of the asymptotic estimate (4) in the theorem is shown in the final section.

\section{The Dyadic SetTing}

We begin by proving the following dyadic version of the factorization theorem. This version is stated for the collection $\mathcal{D}\left(Q_{0}\right)$ of all dyadic subcubes of an arbitrary, fixed cube $Q_{0}$ in $\mathbb{R}^{n}$ : that is, all those cubes obtained by dividing $Q_{0}$ into $2^{n}$ congruent cubes of half its length, dividing each of these into $2^{n}$ congruent cubes, and so on. By convention, $Q_{0}$ itself belongs to $\mathcal{D}\left(Q_{0}\right)$.

Lemma 1. Suppose that $w$ satisfies the dyadic $A_{p}$ condition

$$
\sup _{Q \in \mathcal{D}\left(Q_{0}\right)}\left(\frac{1}{|Q|} \int_{Q} w\right)\left(\frac{1}{|Q|} \int_{Q} w^{1 /(1-p)}\right)^{p-1}=1+\varepsilon \leq 1+\varepsilon_{0}
$$

on the cube $Q_{0}$. Let $f=\log w$. Then there exist functions $g, F$, and $G$ on $Q_{0}$ that satisfy both the pointwise identity

$$
f(x)-f_{Q_{0}}=g(x)+F(x)-G(x), \quad x \in Q_{0},
$$

and the estimates

$$
\begin{aligned}
|g| & \leq C_{1} \sqrt{\varepsilon}, \\
\frac{1}{|Q|} \int_{Q} e^{F} & \leq\left(1+C_{1} \sqrt{\varepsilon}\right) \inf _{Q} e^{F}, \quad Q \in \mathcal{D}\left(Q_{0}\right), \\
\frac{1}{|Q|} \int_{Q} e^{G /(p-1)} & \leq\left(1+C_{1} \sqrt{\varepsilon}\right) \inf _{Q} e^{G /(p-1)}, \quad Q \in \mathcal{D}\left(Q_{0}\right) .
\end{aligned}
$$

The constants $C_{1}$ and $\varepsilon_{0}$ depend only on the dimension $n$ and the index $p$.

Essential to the estimates in the lemma is the following measure-theoretic result (see [8], 9], or [11]), which insures that the mean oscillation of the logarithm of a weight is close to 0 when the $A_{p}$ bound of the weight is near the optimal value 1 .

Lemma 2. If the ratio of the arithmetic and geometric means of $w$ on $Q$ satisfies

$$
\left(\frac{1}{|Q|} \int_{Q} w\right) / \exp \left(\frac{1}{|Q|} \int_{Q} \log w\right)=1+\varepsilon<2
$$

and $f=\log w$, then

$$
\frac{1}{|Q|} \int_{Q}\left|f-f_{Q}\right| \leq C_{2} \sqrt{\varepsilon}
$$


This result holds on each single cube (and, in fact, we may take $C_{2}=32$ ). The form in which we shall apply the estimate is as follows: Let $\|\cdot\|$ and $\|\cdot\|_{*}$ denote the dyadic and full BMO seminorms, i.e.,

$$
\|f\|=\sup _{Q \in \mathcal{D}\left(Q_{0}\right)} \frac{1}{|Q|} \int_{Q}\left|f-f_{Q}\right| \text { and }\|f\|_{*}=\sup _{Q \subset \mathbb{R}^{n}} \frac{1}{|Q|} \int_{Q}\left|f-f_{Q}\right| .
$$

When $w$ satisfies the dyadic $A_{p}$ condition (5), then Jensen's inequality and (10) insure that $\|\log w\| \leq C_{2} \sqrt{\varepsilon}$; likewise, when $A_{p}(w)=1+\varepsilon$, then $\|\log w\|_{*} \leq C_{2} \sqrt{\varepsilon}$.

The following proof of the dyadic version of the factorization theorem combines an iterative Calderón-Zygmund decomposition singling out those cubes on which the mean oscillation of $f$ is large with the bound obtained from Lemma 24

Proof of Lemma国. Fix $Q_{0}$, set $f=\log w$ and $\lambda=2^{n}\|f\|$. Let $\mathcal{G}^{0}=\left\{Q_{0}\right\}$. Define

$$
\mathcal{G}^{1}=\left\{Q_{j} \in \mathcal{D}\left(Q_{0}\right):\left|f_{Q_{j}}-f_{Q_{0}}\right|>\lambda, Q_{j} \text { maximal }\right\}
$$

and, inductively,

$$
\mathcal{G}^{m+1}=\left\{Q_{j} \in \mathcal{D}(Q): Q \in \mathcal{G}^{m},\left|f_{Q_{j}}-f_{Q}\right|>\lambda, Q_{j} \text { maximal }\right\} .
$$

Write $\mathcal{G}=\bigcup_{m=0}^{\infty} \mathcal{G}^{m}$ and let $\Omega^{m}$ be the union of the cubes in $\mathcal{G}^{m}$. By construction, $\Omega^{m+1} \subseteq \Omega^{m} \subseteq \cdots \subseteq \Omega^{0}$. For $Q$ in $\mathcal{G}^{m+1}$, let $\widetilde{Q}$ denote the unique cube in $\mathcal{G}^{m}$ containing $Q$.

Now, maximality in the selection criteria (11) and (12) and standard BMO estimates give rise to the mean-value inequality

$$
\lambda<\left|f_{Q}-f_{\widetilde{Q}}\right| \leq 2 \lambda, \quad Q \in \bigcup_{m=1}^{\infty} \mathcal{G}^{m} .
$$

They also lead to the relative density estimate

$$
\left|Q \cap \Omega^{m+1}\right| \leq 2^{-n}|Q|, \quad Q \in \mathcal{G}^{m},
$$

which is valid for each non-negative integer $m$. Summing this last estimate over the cubes in $\mathcal{G}^{m}$ and iterating leads to the bound

$$
\left|\Omega^{m}\right| \leq 2^{-m n}\left|\Omega^{0}\right| \text {. }
$$

Furthermore, differentiation of the Lebesgue integral —in conjunction with (11) and (12) - yields the pointwise estimate

$$
\left|f(x)-\sum_{Q_{j} \in \mathcal{G}^{m}} f_{Q_{j}} \chi_{Q_{j}}(x)\right| \leq \lambda, \quad x \in \Omega^{m} \backslash \Omega^{m+1},
$$

which is also valid for each non-negative $m$. Hence, when we set

$$
g(x)=f(x)-f_{Q_{0}}-\sum_{m=1}^{\infty} \sum_{Q_{j} \in \mathcal{G}^{m}}\left(f_{Q_{j}}-f_{\widetilde{Q}_{j}}\right) \chi_{Q_{j}}(x),
$$

then $|g| \leq \lambda$ a.e. on $Q_{0}$ 古 The bound $\lambda=2^{n}\|f\| \leq 2^{n} C_{2} \sqrt{\varepsilon}$ from Lemma 2 then gives the desired estimate (7) for $g$.

\footnotetext{
${ }^{4}$ The argument follows [6] and [4] closely, with modifications introduced to get around the fact that the proof for the dyadic model case in [4 pp. 360-361] only leads to $A_{1}$ factors with bounds which are at least 2 , even when the $A_{p}$ bound of the weight to be factored is nearly 1 .

${ }^{5}$ Note that the intersection $\bigcap_{m} \Omega^{m}$ is a set of measure zero within $Q_{0}$, on account of (15). So it suffices to verify the bound for $g$ on $\Omega^{m} \backslash \Omega^{m+1}$ separately for each non-negative $m$, and this follows from (16).
} 
Next, to obtain suitable dyadic $A_{1}$ factors of $w$, split the double sum in (17) according to the sign of the difference $f_{Q_{j}}-f_{\widetilde{Q}_{j}}$. That is, let

$$
f(x)-f_{Q_{0}}=g(x)+F(x)-G(x),
$$

where

$$
F(x)=\sum_{m=1}^{\infty} \sum_{Q_{j} \in \mathcal{G}^{m}}\left(f_{Q_{j}}-f_{\widetilde{Q}_{j}}\right)^{+} \chi_{Q_{j}}(x)
$$

and

$$
G(x)=\sum_{m=1}^{\infty} \sum_{Q_{j} \in \mathcal{G}^{m}}\left(f_{\widetilde{Q}_{j}}-f_{Q_{j}}\right)^{+} \chi_{Q_{j}}(x) .
$$

It is important to note that the functions $F$ and $G$ defined in (18) and (19) are non-negative; where they are positive, their value must, by (13), exceed $\lambda$. For later purposes, we also wish to express $F$ and $G$ as sums over all the dyadic subcubes of $Q_{0}$, not just over those where the mean oscillation of $f$ is large. Thus, we write

$$
F(x)=\sum_{Q_{k} \in \mathcal{D}\left(Q_{0}\right)} a_{k} \chi_{Q_{k}}(x)
$$

and

$$
G(x)=\sum_{Q_{k} \in \mathcal{D}\left(Q_{0}\right)} b_{k} \chi_{Q_{k}}(x) .
$$

In (20), for example, whenever $Q_{k} \notin \bigcup_{m=1}^{\infty} \mathcal{G}^{m}$ or whenever $Q_{k} \in \bigcup_{m=1}^{\infty} \mathcal{G}^{m}$ but $f_{Q_{k}}-f_{\widetilde{Q}_{k}} \leq \lambda$, then $a_{k}=0$; otherwise, $a_{k}=f_{Q_{k}}-f_{\widetilde{Q}_{k}}$. A similar interpretation applies to the coefficients $b_{k}$.

In light of Lemma 2, it suffices to show that the dyadic $A_{1}$ bounds of $\exp F$ and $\exp [G /(p-1)]$ do not exceed $1+C \lambda$, provided that $\lambda=2^{n}\|f\|$ is suitably small. This means we must show that

$$
\frac{1}{|Q|} \int_{Q} e^{F} \leq(1+C \lambda) \inf _{Q} e^{F}
$$

and

$$
\frac{1}{|Q|} \int_{Q} e^{G /(p-1)} \leq(1+C \lambda) \inf _{Q} e^{G /(p-1)}
$$

for all $Q \in \mathcal{D}\left(Q_{0}\right)$. To prove this we now consider three cases.

Case I: The initial cube. We first verify (22) in the case when $Q=Q_{0}$, the original cube. In this case, $\inf _{Q} F=0$, for the choice of $\lambda$ in the stopping-time argument insures that the set $\Omega^{0} \backslash \Omega^{1}$ has positive measure; see (15). Changing variables in the standard integral formula $\int_{Q}\left(e^{F}-1\right)=\int_{0}^{\infty} e^{t}|\{x \in Q: F(x)>t\}| d t$ leads to the equation

$$
\frac{1}{|Q|} \int_{Q} e^{F}=1+\frac{\lambda}{|Q|} \int_{0}^{\infty}\left|E_{\tau}\right| e^{\lambda \tau} d \tau
$$

in which

$$
E_{\tau}=\{x \in Q: F(x)>\lambda \tau\}
$$


Estimating the dyadic $A_{1}$ bound of $\exp F$ then reduces to estimating the size of the set $E_{\tau}$. But condition (13) insures that $E_{\tau} \subseteq \Omega^{1}$, when $0 \leq \tau<2$, and, in general, that $E_{\tau} \subseteq \Omega^{k}$, when $2(k-1) \leq \tau<2 k$ (for each $k$ in $\mathbb{N}$ ). Thus, by (15)) and (24),

$$
\frac{1}{|Q|} \int_{Q} e^{F} \leq 1+2 \lambda \sum_{k=1}^{\infty} \frac{\left|\Omega^{k}\right|}{|Q|} e^{2 \lambda k} \leq 1+2 \lambda \sum_{k=1}^{\infty} 2^{-n k} e^{2 \lambda k} .
$$

The latter sum is less than 2 , when $\lambda=2^{n}\|f\|$ is sufficiently small. Consequently, $|Q|^{-1} \int_{Q} e^{F} \leq 1+4 \lambda$, which is (22) for $Q=Q_{0}$.

Case II: A cube with a large jump in mean value. Suppose now that $Q \in \mathcal{G}^{m}$ for some positive $m$ and that $f_{Q}-f_{\widetilde{Q}}>\lambda .6$ Then

$$
\left(\inf _{Q} e^{F}\right)^{-1} \frac{1}{|Q|} \int_{Q} e^{F}=\frac{1}{|Q|} \int_{Q} e^{F-\inf _{Q} F}=1+\frac{\lambda}{|Q|} \int_{0}^{\infty}\left|\widetilde{E}_{\tau}\right| e^{\lambda \tau} d \tau,
$$

where

$$
\widetilde{E}_{\tau}=\left\{x \in Q: F(x)-\inf _{Q} F>\lambda \tau\right\} .
$$

In analogy to the first case, we find from (13) and (16) that $\widetilde{E}_{\tau} \subset Q \cap \Omega^{m+k}$, when $2(k-1) \leq \tau<2 k$ (for each $k$ in $\mathbb{N}$ ). So for $\tau$ in this range, $\left|\widetilde{E}_{\tau}\right| \leq 2^{-n k}|Q|$, from which the desired estimate (22) once again follows.

Case III: Cubes with no large jump in the mean. In Case I, we considered $Q_{0}$; in Case II, we treated those dyadic cubes $Q$ within $Q_{0}$ for which $f_{Q}-f_{\widetilde{Q}}>\lambda$. To handle the remaining case efficiently, we first introduce a bit of further notation: for each proper dyadic subcube $Q$ of $Q_{0}$, let $\widetilde{Q}$ denote the minimal cube in $\mathcal{G}$ that strictly contains it] and set

$$
\begin{aligned}
\mathcal{P}(Q) & =\left\{Q_{j} \in \mathcal{D}(Q): f_{Q_{j}}-f_{\widetilde{Q}}>\quad \lambda, Q_{j} \text { maximal }\right\}, \\
\mathcal{N}(Q) & =\left\{Q_{j} \in \mathcal{D}(Q): f_{Q_{j}}-f_{\widetilde{Q}}<-\lambda, Q_{j} \text { maximal }\right\} .
\end{aligned}
$$

Note that the union of $\mathcal{P}(Q)$ and $\mathcal{N}(Q)$ is exactly the set of the cubes in $\bigcup_{m=1}^{\infty} \mathcal{G}^{m}$ that lie within $Q$. In this notation, the remaining case now consists of proving (22) on each dyadic cube $Q$ for which $Q \notin \mathcal{P}(Q)$.

Fix such a cube $Q$. To estimate $\int_{Q} \exp F$ we split $Q$ into the union of its subcubes in $\mathcal{P}(Q)$ and the complement of this union. On the one hand, if $Q_{j} \in \mathcal{P}(Q)$, then $\widetilde{Q}_{j}=\widetilde{Q} ;$ Case II then applies, so that

$$
\int_{Q_{j}} e^{F} \leq(1+4 \lambda)\left(\inf _{Q_{j}} e^{F}\right)\left|Q_{j}\right|
$$

But $\inf _{Q_{j}} F=\inf _{\widetilde{Q}} F+\left(f_{Q_{j}}-f_{\widetilde{Q}_{j}}\right)$, hence

$$
\lambda<\inf _{Q_{j}} F-\inf _{\widetilde{Q}} F=f_{Q_{j}}-f_{\widetilde{Q}_{j}} \leq 2 \lambda,
$$

\footnotetext{
${ }^{6}$ Unlike in (12), the sign of the difference is important here.

${ }^{7}$ That is, $\widetilde{Q}=\bigcap\left\{Q_{j} \in \mathcal{G}: Q \subset Q_{j}\right\}$. This is consistent with the earlier notation, in which $Q \in \mathcal{G}^{m+1}$ and $\widetilde{Q} \in \mathcal{G}^{m}$.
} 
by (13). On the other hand, on the complement in $Q$ of $\bigcup Q_{j}$ the value of $F$ is exactly $\inf _{\widetilde{Q}} F$. All together, then,

$$
\begin{aligned}
\int_{Q} e^{F} & \leq(1+4 \lambda) \sum_{Q_{j} \in \mathcal{P}(Q)}\left(\inf _{Q_{j}} e^{F}\right)\left|Q_{j}\right|+\left(\inf _{\widetilde{Q}} e^{F}\right)\left|Q \backslash \bigcup_{Q_{j} \in \mathcal{P}(Q)} Q_{j}\right| \\
& \leq(1+4 \lambda) e^{2 \lambda}\left(\inf _{\widetilde{Q}} e^{F}\right) \sum_{Q_{j} \in \mathcal{P}(Q)}\left|Q_{j}\right|+\left(\inf _{\widetilde{Q}} e^{F}\right)\left|Q \backslash \bigcup_{Q_{j} \in \mathcal{P}(Q)} Q_{j}\right| \\
& \leq(1+4 \lambda) e^{2 \lambda}\left(\inf _{\widetilde{Q}} e^{F}\right)|Q| .
\end{aligned}
$$

Since $\inf _{\widetilde{Q}} F \leq \inf _{Q} F$, the bound (22) thus also holds for the cubes $Q$ in this, the last case.

The justification of the dyadic $A_{1}$ bound (23) is similar, with $G /(p-1)$ in place

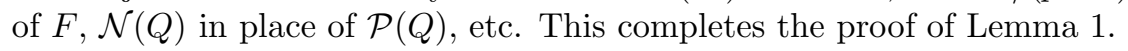

\section{The General Setting}

The proof of the theorem follows the argument in 4, pp. 361-364], except for certain technical modifications which are introduced to keep all bounds as small as possible. For completeness, the full proof is given here. Let $S_{N}$ be the cube $\left\{x \in \mathbb{R}^{n}:\left|x_{i}\right| \leq 2^{N}, 1 \leq i \leq n\right\}$.

Lemma 3. Suppose that $w \in A_{p}$ and that $A_{p}(w)=1+\varepsilon<1+\varepsilon_{0}$. Let $f=\log w$. For each natural number $N$ there exist functions $g_{N}, F_{N}$, and $G_{N}$ on the cube $S_{N}$ satisfying both the pointwise identity

$$
f(x)-f_{S_{N}}=g_{N}(x)+F_{N}(x)-G_{N}(x), \quad x \in S_{N},
$$

and the bounds

$$
\begin{aligned}
\left|g_{N}\right| & \leq C_{3} \sqrt{\varepsilon} \\
\frac{1}{|Q|} \int_{Q} e^{F_{N}} & \leq\left(1+C_{3} \sqrt{\varepsilon}\right) \inf _{Q} e^{F_{N}}, \quad Q \subseteq S_{N}, \\
\frac{1}{|Q|} \int_{Q} e^{G_{N} /(p-1)} & \leq\left(1+C_{3} \sqrt{\varepsilon}\right) \inf _{Q} e^{G_{N} /(p-1)}, \quad Q \subseteq S_{N} .
\end{aligned}
$$

The constants $C_{3}$ and $\varepsilon_{0}$ depend only on the dimension $n$ and the index $p$.

Note that (28) and 29) are valid for all (not just dyadic) subcubes of $S_{N}$.

Let us first show how this last lemma implies the theorem. The identity (26) can be re-written, after subtracting off the mean value of each side on $S_{0}$, as

$$
\begin{aligned}
f(x)-f_{S_{0}} & =\left[g_{N}(x)-\left(g_{N}\right)_{S_{0}}\right]+\left[F_{N}(x)-\left(F_{N}\right)_{S_{0}}\right]-\left[G_{N}(x)-\left(G_{N}\right)_{S_{0}}\right] \\
& =\tilde{g}_{N}(x)+\tilde{F}_{N}(x)-\tilde{G}_{N}(x) .
\end{aligned}
$$

Then $\left|\tilde{g}_{N}\right| \leq 2 C_{3} \sqrt{\varepsilon}$ a.e. on $S_{N}$, by (27). Taking the logarithm of (28) readily yields a bound on the mean oscillation of $F_{N}$ :

$$
\frac{1}{|Q|} \int_{Q}\left|F_{N}-\left(F_{N}\right)_{Q}\right| \leq \frac{2}{|Q|} \int_{Q}\left(F_{N}-\inf _{Q} F_{N}\right) \leq 2 C_{3} \sqrt{\varepsilon}, \quad Q \subseteq S_{N} .
$$

The same estimate applies to $\tilde{F}_{N}$, since it differs from $F_{N}$ only by an additive constant. The John-Nirenberg inequality in [7] then allows us to convert this statement 
into a bound on the quadratic mean oscillation of $\tilde{F}_{N}$, namely

$$
\frac{1}{|Q|} \int_{Q}\left|\tilde{F}_{N}-\left(\tilde{F}_{N}\right)_{Q}\right|^{2} \leq C^{\prime} \varepsilon, \quad Q \subseteq S_{N} .
$$

Suppose now that $M \leq N$. When $Q=S_{M}$, the last estimate becomes

$$
\frac{1}{\left|S_{M}\right|} \int_{S_{M}}\left|\tilde{F}_{N}\right|^{2} \leq C^{\prime} \varepsilon+\left|\left(\tilde{F}_{N}\right)_{S_{M}}\right|^{2}
$$

To control the right-hand side, form a telescoping sum of mean values:

$$
\left(\tilde{F}_{N}\right)_{S_{M}}=\left(\tilde{F}_{N}\right)_{S_{0}}+\left[\left(\tilde{F}_{N}\right)_{S_{1}}-\left(\tilde{F}_{N}\right)_{S_{0}}\right]+\cdots+\left[\left(\tilde{F}_{N}\right)_{S_{M}}-\left(\tilde{F}_{N}\right)_{S_{M-1}}\right] .
$$

Since $\left|S_{1}\right| /\left|S_{0}\right|=\cdots=\left|S_{M}\right| /\left|S_{M-1}\right|=2^{n}$, the magnitude of each of the $M$ bracketed differences is no more than the fixed quantity $2^{n}\left(C_{3} \sqrt{\varepsilon}\right)$, by (32). In fact, as $\left(\tilde{F}_{N}\right)_{S_{0}}=0$, (34) becomes $\left|\left(F_{N}\right)_{S_{M}}\right| \leq M 2^{n} C_{3} \sqrt{\varepsilon}$. Conditions (33) and (34) together then yield the quadratic bound

$$
\frac{1}{\left|S_{M}\right|} \int_{S_{M}}\left|\tilde{F}_{N}\right|^{2} \leq C^{\prime} \varepsilon+\left(M 2^{n} C_{3} \sqrt{\varepsilon}\right)^{2}<\infty,
$$

which holds uniformly for $N=M, M+1, M+2, \ldots$, and an analogous bound is also valid for $\tilde{G}_{N}$. For each $M$, the sequences $\left\{\tilde{F}_{N}: N \geq M\right\}$ and $\left\{\tilde{G}_{N}: N \geq M\right\}$ are thus bounded in $L^{2}\left(S_{M}\right)$; we have also already seen that $\left\{\tilde{g}_{N}: N \geq M\right\}$ is a bounded sequence in $L^{\infty}\left(S_{N}\right)$. Using a diagonal argument, we may therefore choose a subsequence $N_{j} \rightarrow \infty$, so that $\tilde{F}_{N_{j}} \rightarrow F, \quad \tilde{G}_{N_{j}} \rightarrow G$ weakly in $L^{2}\left(S_{M}\right)$ and so that $\tilde{g}_{N_{j}} \rightarrow g$ in the weak-star topology on $L^{\infty}\left(S_{M}\right)$, with this convergence holding simultaneously for all $M 8$ Moreover, on each cube $S_{M}$ there is a sequence of finite convex combinations $\sum_{j=1}^{J} t_{j} \widetilde{F}_{N_{j}}$, with $t_{j} \geq 0$ and $\sum_{j=1}^{J} t_{j}=1$, that converges to $F$ both in $L^{2}$ and (taking a further subsequence, if necessary) pointwise a.e 9 From (31), then, $f(x)-f_{S_{0}}=g(x)+F(x)-G(x)$, with

$$
|g| \leq C_{3} \sqrt{\varepsilon} \quad \text { a.e. on } \mathbb{R}^{n} \text {. }
$$

To obtain the desired $A_{1}$ bound on $\exp F$, fix an arbitrary cube $Q$ in $\mathbb{R}^{n}$, and choose $M$ so large that $Q \subseteq S_{M}$. Apply Fatou's lemma and Hölder's inequality to the sequence $\left\{\exp \sum_{j=1}^{J} t_{j} \tilde{F}_{N_{j}}: N_{j} \geq M\right\}$ to obtain the bound

$$
\frac{1}{|Q|} \int_{Q} e^{F} \leq \lim \inf _{J \rightarrow \infty} \prod_{j=1}^{J}\left(\frac{1}{|Q|} \int_{Q} e^{\tilde{F}_{N_{j}}}\right)^{t_{j}} \leq\left(1+C_{3} \sqrt{\varepsilon}\right) \inf _{Q} e^{F}
$$

from (28). Set $u=\exp \left[f_{S_{0}}+g+F\right]$. Thanks to (35) and (36), $u \in A_{1}$ and $A_{1}(u) \leq \exp \left[2 C_{3} \sqrt{\varepsilon}\right]\left(1+C_{3} \sqrt{\varepsilon}\right)=1+\mathcal{O}(\sqrt{\varepsilon})$, as desired. The corresponding $A_{1}$ bound for $v=\exp [G /(p-1)]$ follows similarly from (29). The proof of the theorem is now complete.

\footnotetext{
${ }^{8}$ The John-Nirenberg inequality has been invoked to move from uniform boundedness in $L^{1}$ to that in $L^{2}$; otherwise, weak compactness would have only guaranteed the existence of a subsequence converging to a measure.

${ }^{9}$ See Theorem 3.13 in [14] or Theorem V.1.2 in [16]; in the latter work, this result is attributed to S. Mazur.

${ }^{10}$ Suppose that $\left\{\varphi_{J}\right\}$ is a sequence of non-negative, measurable functions that converges a.e. to $\varphi$. What is needed here are both the (standard) $L^{1}$ form of Fatou's lemma, $\int \varphi \leq \liminf \operatorname{lin}_{J} \int \varphi_{J}$, as well as its $L^{\infty}$ form: $\liminf \inf _{J}\left(\inf \varphi_{J}\right) \leq \inf \varphi$; the latter can be verified via a simple proof by contradiction. Recall that we write $\inf \varphi$ for $\operatorname{ess} \inf \varphi$, as indicated in the introduction.
} 
Proof of Lemma 3. We use the averaging procedure of 4 to move from the dyadic version of the theorem (Lemma 1) to the general, local version (Lemma 3). Fix $N$ and assume, without loss of generality, that $f_{S_{N}}=0$. Set $Q_{0}=S_{N+1}$ and $\lambda=2^{n}\|f\|_{*}$. For each $\alpha \in S_{N}$, apply Lemma 1 on $Q_{0}$ to the translate $T_{\alpha} f$ of $f$, where $T_{\alpha} f(x)=f(x-\alpha)$; note that condition (5) holds uniformly for $e^{T_{\alpha} f}$ (in place of $\left.w=e^{f}\right)$ as $\alpha$ varies, due to the assumption that $A_{p}\left(e^{f}\right)=1+\varepsilon$. The result is

$$
T_{\alpha} f(x)-\left(T_{\alpha} f\right)_{S_{N+1}}=g^{(\alpha)}(x)+F^{(\alpha)}(x)-G^{(\alpha)}(x),
$$

where $g^{(\alpha)}, F^{(\alpha)}$, and $G^{(\alpha)}$ satisfy (7), (8), and (9), respectively 11 Next, for a.e. $x$ within the cube $S_{N}$, we know that

$$
\begin{aligned}
f(x) & =\frac{1}{\left|S_{N}\right|} \int_{S_{N}} T_{-\alpha}\left(T_{\alpha} f\right)(x) d \alpha \\
& =\frac{1}{\left|S_{N}\right|} \int_{S_{N}} T_{-\alpha}\left(g^{(\alpha)}+\left(T_{\alpha} f\right)_{S_{N+1}}+F^{(\alpha)}-G^{(\alpha)}\right)(x) d \alpha \\
& =g_{N}(x)+F_{N}(x)-G_{N}(x),
\end{aligned}
$$

where, in the last line, $g_{N}(x)=\left|S_{N}\right|^{-1} \int_{S_{N}} T_{-\alpha}\left(g^{(\alpha)}+\left(T_{\alpha} f\right)_{S_{N+1}}\right)(x) d \alpha$ and $F_{N}(x)=\left|S_{N}\right|^{-1} \int_{S_{N}} T_{-\alpha}\left(F^{(\alpha)}\right)(x) d \alpha$, and where $G_{N}$ is defined analogously to $F_{N}$. Now, since $f$ is in BMO, then

$$
\left|\left(T_{\alpha} f\right)_{S_{N+1}}\right| \leq\left|\left(T_{\alpha} f\right)_{S_{N+1}}-f_{S_{N+1}}\right|+\left|f_{S_{N+1}}-f_{S_{N}}\right|+\left|f_{S_{N}}\right| \leq c_{n} \sqrt{\varepsilon},
$$

as follows from (10) and the assumption $f_{S_{N}}=0.12$ The uniform boundedness of $g^{(\alpha)}$ in (7) then insures that $\left|g_{N}\right| \leq C_{3} \sqrt{\varepsilon}$ a.e. on $S_{N}$. In addition, the expansion (20) guarantees that there are non-negative coefficient functions $a_{k}^{(\alpha)}$, depending measurably on $\alpha 13$ such that

$$
F^{(\alpha)}(x)=\sum_{Q_{k} \in \mathcal{D}\left(S_{N+1}\right)} a_{k}^{(\alpha)} \chi_{Q_{k}}(x) .
$$

Note that this sum runs over $\mathcal{D}\left(S_{N+1}\right)$, a fixed, countable collection of cubes which is indexed by $k$ and independent of $\alpha$; as in $₫ 2$, each coefficient $a_{k}^{(\alpha)}$ is either 0 or a number between $\lambda$ and $2 \lambda$. Condition (21) leads to a similar representation for $G^{(\alpha)}$.

It remains to show that $F_{N}$ satisfies the desired $A_{1}$ estimate on $S_{N}$. Fix an arbitrary cube $Q$ within $S_{N}$. Our goal is to show (28), i.e.,

$$
\frac{1}{|Q|} \int_{Q} e^{F_{N}} \leq\left(1+C_{3} \sqrt{\varepsilon}\right) \inf _{Q} e^{F_{N}}
$$

\footnotetext{
${ }^{11}$ Symbols such as $\mathcal{G}^{m,(\alpha)}, \mathcal{G}^{(\alpha)}$, and $\Omega^{m,(\alpha)}$ will likewise denote the sets within $Q_{0}$ obtained when $f$ is replaced by its translate $T_{\alpha} f$ in the definitions of $\mathcal{G}^{m}, \mathcal{G}$, and $\Omega^{m}$ in $\$ 2$

${ }^{12}$ Compare the bound obtained from 34 .

${ }^{13}$ Choose a dyadic subcube $Q_{k}$ of $Q_{0}$, with $\left|Q_{k}\right|=2^{-n}\left|Q_{0}\right|$. By definition, the coefficient $a_{k}^{(\alpha)}$ satisfies $a_{k}^{(\alpha)}=\left[\left(T_{\alpha} f\right)_{Q_{k}}-\left(T_{\alpha} f\right)_{Q_{0}}\right] \chi_{E_{k}}$, where $E_{k}=\left\{\alpha \in S_{N}:\left[\left(T_{\alpha} f\right)_{Q_{k}}-\left(T_{\alpha} f\right)_{Q_{0}}\right]>\lambda\right\}$. Since $f \in L^{1}\left(S_{N+2}\right)$, then [ $\left.\cdots\right]$ is a continuous function of $\alpha$, and $E_{k}$ is consequently an open set within $S_{N}$. This proves the measurability in $\alpha$ of the coefficient functions $a_{k}^{(\alpha)}$ associated to each first-generation subcube $Q_{k}$ of $Q_{0}$. The argument for cubes of a later generation within $\mathcal{D}\left(Q_{0}\right)$ is analogous.
} 
To reach this we will make a number of reductions. First, on the cube $Q$, write $F^{(\alpha)}=F_{1}^{(\alpha)}+F_{2}^{(\alpha)}$, with

$$
F_{1}^{(\alpha)}=\sum_{\ell\left(Q_{k}\right) \geq \ell(Q)} a_{k}^{(\alpha)} \chi_{Q_{k}}, \quad F_{2}^{(\alpha)}=\sum_{\ell\left(Q_{k}\right)<\ell(Q)} a_{k}^{(\alpha)} \chi_{Q_{k}},
$$

where $\ell(Q)$ denotes the side-length of $Q$. Note that only finitely many terms enter into the first sum. Next, define the averaged forms

$$
F_{N, 1}(x)=\frac{1}{\left|S_{N}\right|} \int_{S_{N}} F_{1}^{(\alpha)}(x+\alpha) d \alpha, \quad F_{N, 2}(x)=\frac{1}{\left|S_{N}\right|} \int_{S_{N}} F_{2}^{(\alpha)}(x+\alpha) d \alpha ;
$$

thus, $F_{N}=F_{N, 1}+F_{N, 2}$. On account of Lemma 1, to prove (37) it suffices to show the two bounds

$$
\sup _{Q} F_{N, 1}-\inf _{Q} F_{N, 1} \leq C \lambda
$$

and

$$
\frac{1}{|Q|} \int_{Q} e^{F_{N, 2}} \leq(1+C \lambda) \inf _{Q} e^{F_{N, 2}}
$$

where $\lambda=2^{n}\|f\|_{*}$.

Now, (38) is a consequence of the following Lipschitz estimate14 on the contribution to $F_{N}$ of the terms arising from cubes of a fixed size:

Lemma 4. Let

$$
\hat{F}_{j}(x)=\frac{1}{\left|S_{N}\right|} \int_{S_{N}} \sum_{\ell\left(Q_{k}\right)=2^{-j} \ell\left(S_{N}\right)} a_{k}^{(\alpha)} \chi_{Q_{k}}(x),
$$

so that $F_{N}(x)=\sum_{j=0}^{\infty} \hat{F}_{j}(x)$. If $\sup _{1 \leq i \leq n}\left|x_{i}-y_{i}\right| \leq 2^{-j} \ell\left(S_{N}\right)$, then

$$
\left|\hat{F}_{j}(x)-\hat{F}_{j}(y)\right| \leq \frac{C_{4} 2^{j}\|f\|_{*}}{\ell\left(S_{N}\right)}|x-y|
$$

with $C_{4}$ dependent only on the dimension $n$ (and, in particular, not on $j$ ).

In fact, if $x, y \in Q$ and $r$ is the integer satisfying $2^{-r-1} \ell\left(S_{N}\right)<\ell(Q) \leq 2^{-r} \ell\left(S_{N}\right)$, then $\sup _{1 \leq i \leq n}\left|x_{i}-y_{i}\right| \leq 2^{-r} \ell\left(S_{N}\right)$. Hence

$$
\left|F_{N, 1}(x)-F_{N, 1}(y)\right| \leq \sum_{j=0}^{r}\left|\hat{F}_{j}(x)-\hat{F}_{j}(y)\right| \leq C_{4}\|f\|_{*} \sum_{j=0}^{r} 2^{j} \frac{|x-y|}{\ell\left(S_{N}\right)} .
$$

The latter sum is no more than $2 \sqrt{n}$, so that (38) holds.

What about (39)? We can, in fact, further simplify the right-hand side there by noting that $F_{N, 2} \geq 0$. As for the left-hand side, from Jensen's inequality and Fubini's theorem it follows that

$$
\begin{aligned}
\frac{1}{|Q|} \int_{Q} e^{F_{N, 2}} & =\frac{1}{|Q|} \int_{Q} \exp \left[\frac{1}{\left|S_{N}\right|} \int_{S_{N}} T_{-\alpha}\left(F_{2}^{(\alpha)}\right)(x) d \alpha\right] d x \\
& \leq \frac{1}{|Q|\left|S_{N}\right|} \int_{Q} \int_{S_{N}} \exp \left[T_{-\alpha}\left(F_{2}^{(\alpha)}\right)(x)\right] d \alpha d x \\
& =\frac{1}{\left|S_{N}\right|} \int_{S_{N}} \frac{1}{|Q|} \int_{Q+\alpha} \exp \left(F_{2}^{(\alpha)}\right)(y) d y d \alpha
\end{aligned}
$$

\footnotetext{
${ }^{14}$ This is Lemma 3.2 in 4 .
} 
For the proof of (37), it thus suffices to obtain a suitable estimate on the inner integral in the last line, i.e., to show that

$$
\frac{1}{|Q|} \int_{Q+\alpha} \exp \left(F_{2}^{(\alpha)}\right)(y) d y=1+\mathcal{O}(\lambda)
$$

uniformly for all $\alpha \in S_{N}$. The last integral average can be written, as in (24), in the form

$$
1+\frac{\lambda}{|Q|} \int_{0}^{\infty}\left|E_{\tau, 2}^{(\alpha)}\right| e^{\lambda \tau} d \tau
$$

where

$$
E_{\tau, 2}^{(\alpha)}=\left\{y \in Q+\alpha: F_{2}^{(\alpha)}(y)>\lambda \tau\right\}
$$

But $Q+\alpha$ is contained within a union of $2^{n}$ dyadic subcubes of $S_{N+1}$, each having side-length less than twice that of $Q$. Applying the construction in 2 to each of these subcubes and summing leads to the estimate $\left|E_{\tau, 2}^{(\alpha)}\right| \leq c_{n} 2^{-n k}$, when $2(k-1) \leq \tau$. The bound (41) then follows from writing $\int_{0}^{\infty}(\cdots) d \tau$ as the sum $\sum_{k=1}^{\infty} \int_{2(k-1)}^{2 k}(\cdots) d \tau$. The proof of estimate (29) for $G_{N}$ is similar. This settles the last remaining step in the proof of the lemma, and the factorization theorem is thus complete.

\section{Sharpness of the ASymptotic estimate}

That the square root is the sharp power in the theorem follows from considering a step function $w$ with the value $1+\sqrt{\varepsilon}$ on one side and $1-\sqrt{\varepsilon}$ on the other side of a hyperplane in $\mathbb{R}^{n}$. This weight satisfies $A_{p}(w)=1+\mathcal{O}(\varepsilon)$, although, as we shall presently show, regardless of how it is factored into a quotient of $A_{1}$ weights, at least one of its factors must have an $A_{1}$ bound exceeding $1+\mathcal{O}(\sqrt{\varepsilon})$.

Proposition. Let $w$ be the step function taking the value $1+\sqrt{\varepsilon}$ in $\mathbb{R}_{+}^{n}$ and $1-\sqrt{\varepsilon}$ in $\mathbb{R}_{-}^{n}$. Suppose that $w=u v^{1-p}$ for $A_{1}$ weights $u$ and $v$. Then

$$
A_{p}(w) \leq 1+c \varepsilon
$$

although

$$
\max \left[A_{1}(u), A_{1}(v)\right] \geq 1+c^{-1} \sqrt{\varepsilon} .
$$

The constant $c$ depends only on the index $p$.

Proof. For simplicity, we first show this in the case $p=2$. Divide the unit cube $Q=[-1 / 2,1 / 2]^{n}$ in half, with $I=Q \cap \mathbb{R}_{+}^{n}$ and $J=Q \cap \mathbb{R}_{-}^{n}$. A calculation shows that the $A_{2}$ bound of the given weight $w$ is achieved when the averages of $w$ and $w^{-1}$ are formed symmetrically over $Q$, in which case

$$
A_{2}(w)=\left[\frac{1+\sqrt{\varepsilon}}{2}+\frac{1-\sqrt{\varepsilon}}{2}\right]\left[\frac{1}{2(1+\sqrt{\varepsilon})}+\frac{1}{2(1-\sqrt{\varepsilon})}\right]=\frac{1}{1-\varepsilon}=1+\mathcal{O}(\varepsilon) .
$$

Suppose that $w=u / v$ for the pair of $A_{1}$ weights $u, v$. If $A_{1}(u) \leq 1+\sqrt{\varepsilon} / 4$, then

$$
\int_{Q} v \geq\left[\frac{1}{1+\sqrt{\varepsilon}}+\frac{1}{1-\sqrt{\varepsilon}}\right] \min \left[\int_{I} u, \int_{J} u\right] \geq \frac{2}{1-\varepsilon} \frac{1}{1+\sqrt{\varepsilon} / 2} \int_{I} u,
$$


where the last step is a simple consequence of the assumed $A_{1}$ bound on $u$ In addition,

$$
\inf _{Q} v \leq \inf _{I} v=\frac{1}{1+\sqrt{\varepsilon}} \inf _{I} u \leq \frac{2}{1+\sqrt{\varepsilon}} \int_{I} u
$$

Hence

$$
A_{1}(v) \geq \frac{\int_{Q} v}{\inf _{Q} v} \geq \frac{1+\sqrt{\varepsilon}}{(1-\varepsilon)(1+\sqrt{\varepsilon} / 2)} \geq \frac{1}{1-\sqrt{\varepsilon} / 2} \geq 1+\frac{1}{2} \sqrt{\varepsilon}
$$

When $p>2$, the argument is similar: if $A_{1}(u) \leq 1+\sqrt{\varepsilon} / 4$ and $v^{p-1}=u / w$, then the above estimates show that $A_{1}(v) \geq(1+\sqrt{\varepsilon} / 2)^{1 /(p-1)} \geq 1+\sqrt{\varepsilon} / 2$. When $p<2$, it is easier to begin with an $A_{1}$ weight $v$ and to set $u=w v^{\bar{p}-1}$. In this case, if $A_{1}(v) \leq 1+\sqrt{\varepsilon} / C_{p}$, then $A_{1}(u) \geq(1+\sqrt{\varepsilon})^{-1}\left(1+2 \sqrt{\varepsilon} / C_{p}\right)^{1-p}$; the last quantity exceeds $1+c^{-1} \sqrt{\varepsilon}$, provided that $C_{p}$ is sufficiently large. This completes the proof of the proposition.

\section{REFERENCES}

1. R. R. Coifman and C. Fefferman, Weighted norm inequalities for maximal functions and singular integrals, Studia Math. 51 (1974), 241-250. MR 50:10670

2. R. R. Coifman, J. Rubio de Francia, and P. Jones, Constructive decomposition of BMO functions and factorization of $A_{p}$ weights, Proc. Amer. Math. Soc. 87 (1983), 675-676. MR 84c:42031

3. J. García-Cuerva and J. L. Rubio de Francia, Weighted norm inequalities and related topics, North-Holland, Amsterdam, New York, and Oxford, 1985. MR 87d:42043

4. J. Garnett and P. Jones, BMO from dyadic BMO, Pacific J. Math. 99 (1982), 351-371. MR 85d:42021

5. P. J. Holden, Extension theorems for functions of vanishing mean oscillation, Pacific J. Math. 142 (1990), 277-295. MR 91c:42027

6. P. Jones, Factorization of $A_{p}$ weights, Ann. of Math. 111 (1980), 511-530. MR 82b:46035

7. F. John and L. Nirenberg, On functions of bounded mean oscillation, Comm. Pure Appl. Math. 14 (1961), 415-426. MR 24:A1348

8. M. B. Korey, Ideal weights: doubling and absolute continuity with asymptotically optimal bounds, Ph.D. Thesis, University of Chicago, 1995.

9. M. B. Korey, Ideal weights: asymptotically optimal versions of doubling, absolute continuity, and mean oscillation, J. Fourier Anal. Appl. 4 (1998), 491-519. CMP 99:05

10. B. Muckenhoupt, Weighted norm inequalities for the Hardy maximal function, Trans. Amer. Math. Soc. 165 (1972), 207-226. MR 45:2461

11. A. Politis, Sharp results on the relation between weight spaces and BMO, Ph.D. Thesis, University of Chicago, 1995.

12. J. Rubio de Francia, Factorization and extrapolation of weights, Bull. Amer. Math. Soc. 7 (1982), 393-395. MR 83i:42016

13. J. Rubio de Francia, Factorization theory and $A_{p}$ weights, Amer. J. Math. 106 (1984), 533547. MR 86a:47028a

14. W. Rudin, Functional analysis, McGraw-Hill, New York, 1973. MR 51:1315

15. E. M. Stein, Harmonic analysis: Real-variable methods, orthogonality, and oscillatory integrals, Princeton Univ. Press, Princeton, NJ, 1993. MR 95c:42002

16. K. Yosida, Functional analysis, Grundlehren Math. Wiss., vol. 123, Springer-Verlag, Berlin, Heidelberg, and New York, 1965. MR 31:5054

Institut für Mathematik, Universität Potsdam, 14415 Potsdam, Germany

E-mail address: mike@math.uni-potsdam.de

\footnotetext{
${ }^{15}$ When $|Q|^{-1} \int_{Q} u=(1+\delta) \inf _{Q} u$ and $Q$ is divided into two halves $I, J$ of equal measure, then a simple calculation shows that $\left(\int_{I} u\right) /\left(\int_{J} u\right) \leq 1+2 \delta$ (see, e.g., [9] $\S 2$, Cor. 7]).
} 